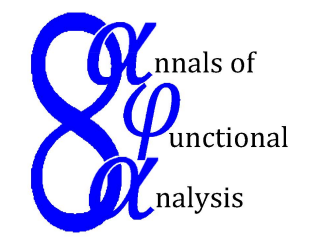

Ann. Funct. Anal. 6 (2015), no. 2, 173-183

http://doi.org/10.15352/afa/06-2-15

ISSN: 2008-8752 (electronic)

http://projecteuclid.org/afa

\title{
SPECTRAL PROPERTIES AND RESTRICTIONS OF BOUNDED LINEAR OPERATORS
}

\author{
C. CARPINTERO ${ }^{1 *}$, E. ROSAS ${ }^{1}$, J. RODRIGUEZ ${ }^{2}$, D. MUÑOZ ${ }^{3}$ AND K. ALCALÁ ${ }^{3}$ \\ Communicated by M. S. Moslehian
}

\begin{abstract}
Assume $T \in L(X)$ is a bounded linear operator on a Banach space $X$, and that $T_{n}$ is a restriction of $T$ on $R\left(T^{n}\right)=T^{n}(X)$. In general, almost nothing can be said concerning the relationship between the spectral properties of $T$ and $T_{n}$. However, under some conditions, it is shown that several spectral properties introduced recently are the same for $T$ and $T_{n}$.
\end{abstract}

\section{INTRODUCTION AND PRELIMINARIES}

Throughout this paper $L(X)$ denotes the algebra of all bounded linear operators acting on an infinite-dimensional complex Banach space $X$. For $T \in L(X)$, we denote by $N(T)$ the null space of $T$ and by $R(T)=T(X)$ the range of $T$. We denote by $\alpha(T):=\operatorname{dim} N(T)$ the nullity of $T$ and by $\beta(T):=\operatorname{codim} R(T)=$ $\operatorname{dim} X / R(T)$ the defect of $T$. Other two classical quantities in operator theory are the ascent $p=p(T)$ of an operator $T$, defined as the smallest non-negative integer $p$ such that $N\left(T^{p}\right)=N\left(T^{p+1}\right)$ ( if such an integer does not exist, we put $p(T)=$ $\infty)$, and the descent $q=q(T)$, defined as the smallest non-negative integer $q$ such that $R\left(T^{q}\right)=R\left(T^{q+1}\right.$ ) (if such an integer does not exist, we put $q(T)=\infty$ ). It is well known that if $p(T)$ and $q(T)$ are both finite then $p(T)=q(T)$. Furthermore, $0<p(\lambda I-T)=q(\lambda I-T)<\infty$ if and only if $\lambda$ is a pole of the resolvent, see [15, Prop. 50.2]. An operator $T \in L(X)$ is said to be Fredholm (resp. upper semi-Fredholm, lower semi-Fredholm), if $\alpha(T), \beta(T)$ are both finite (resp. $R(T)$ closed and $\alpha(T)<\infty, \beta(T)<\infty)$. T $T L L(X)$ is said to be semi-Fredholm if $T$ is either an upper semi-Fredholm or a lower semi-Fredholm operator. If $T$ is

Date: Received: Jan. 2, 2014; Accepted: Jul. 24, 2014.

${ }^{*}$ Corresponding author.

2010 Mathematics Subject Classification. Primary 47A10; Secondary 47A11, 47A53, 47A55.

Key words and phrases. semi Fredholm operators, poles of the resolvent, single valued extension property, ascent, descent. 
semi-Fredholm then the index of $T$ defined by ind $T:=\alpha(T)-\beta(T)$. Other two important classes of operators in Fredholm theory are the classes of upper/lower semi-Browder operators. These classes are defined as follows: $T \in L(X)$ is said to be Browder (resp. upper semi-Browder, lower semi-Browder) if $T$ is a Fredholm (resp. upper semi-Fredholm, lower semi-Fredholm) operator and both $p(T)$ and $q(T)$ are finite (resp. $p(T)<\infty, q(T)<\infty$ ). A operator $T \in L(X)$ is said to be upper semi-Weyl (resp. lower semi-Weyl) if $T$ is upper Fredholm (resp. lower semi-Fredholm) operator and index ind $T \leq 0$ (resp. ind $T \geq 0$ ). $T \in L(X)$ is said to be Weyl if $T$ is both upper and lower semi-Weyl, i.e. $T$ is a Fredholm operator having index 0. The Browder spectrum and the Weyl spectrum are defined, respectively, by

$$
\sigma_{\mathrm{b}}(T):=\{\lambda \in \mathbb{C}: \lambda I-T \text { is not Browder }\},
$$

and

$$
\sigma_{\mathrm{w}}(T):=\{\lambda \in \mathbb{C}: \lambda I-T \text { is not Weyl }\} .
$$

Since every Browder operator is Weyl, $\sigma_{\mathrm{w}}(T) \subseteq \sigma_{\mathrm{b}}(T)$. Analogously, the upper semi-Browder spectrum and the upper semi-Weyl spectrum are defined by

$$
\sigma_{\mathrm{ub}}(T):=\{\lambda \in \mathbb{C}: \lambda I-T \text { is not upper semi-Browder }\},
$$

and

$$
\sigma_{\text {uw }}(T):=\{\lambda \in \mathbb{C}: \lambda I-T \text { is not upper semi-Weyl }\} .
$$

Given $n \in \mathbb{N}$, we denote by $T_{n}$ the restriction of $T \in L(X)$ on the subspace $R\left(T^{n}\right)=T^{n}(X)$. According [5] and [6], $T \in L(X)$ is said to be semi B-Fredholm (resp. B-Fredholm, upper semi B-Fredholm, lower semi B-Fredholm), if for some integer $n \geq 0$ the range $R\left(T^{n}\right)$ is closed and $T_{n}$, viewed as a operator from the space $R\left(T^{n}\right)$ into itself, is a semi-Fredholm (resp. Fredholm, upper semiFredholm, lower semi-Fredholm) operator. Analogously, $T \in L(X)$ is said to be $B$-Browder (resp. upper semi B-Browder, lower semi B-Browder), if for some integer $n \geq 0$ the range $R\left(T^{n}\right)$ is closed and $T_{n}$ is a Browder (resp. upper semiBrowder, lower semi -Browder) operator. If $T_{n}$ is a semi-Fredholm operator, it follows from [6, Proposition 2.1] that also $T_{m}$ is semi-Fredholm for every $m \geq n$, and ind $T_{m}=\operatorname{ind} T_{n}$. This enables us to define the index of a semi B-Fredholm operator $T$ as the index of the semi-Fredholm operator $T_{n}$. Thus, $T \in L(X)$ is said to be a $B$-Weyl operator if $T$ is a B-Fredholm operator having index 0 . $T \in L(X)$ is said to be upper semi $B$-Weyl (resp. lower semi $B$-Weyl) if $T$ is upper semi B-Fredholm (resp. lower semi B-Fredholm) with index ind $T \leq 0$ (resp. ind $T \geq 0$ ). Note that if $T$ is B-Fredholm then also $T^{*}$ is B-Fredholm with ind $T^{*}=-\operatorname{ind} T$. An operator $T \in L(X)$ is said to be left Drazin invertible (resp. right Drazin invertible) if $p(T)<\infty$ (resp. $q(T)<\infty)$ and $R\left(T^{p(T)+1}\right)$ (resp. $R\left(T^{q(T)}\right)$ ) is closed. $T \in L(X)$ is called Drazin invertible if the ascent and the descent of $T$ are both finite. It is proved in [5, Theorem 3.6] that $T$ is a B-Browder operator (resp. upper semi B-Browder, lower semi B-Browder) if and only if $T$ is a Drazin invertible (resp. left Drazin invertible, right Drazin invertible) operator. 
Another spectra related with semi B-Fredholm operators are defined as follows. The Drazin invertible spectrum is defined by

$$
\sigma_{\mathrm{d}}(T):=\{\lambda \in \mathbb{C}: \lambda I-T \text { is not Drazin invertible }\} .
$$

The $B$-Weyl spectrum is defined by

$$
\sigma_{\mathrm{bw}}(T):=\{\lambda \in \mathbb{C}: \lambda I-T \text { is not B-Weyl }\},
$$

while the $B$-Browder spectrum is defined by

$$
\sigma_{\mathrm{bb}}(T)=\{\lambda \in \mathbb{C}: \lambda I-T \text { is not B-Browder }\} .
$$

Clearly, by [5, Theorem 3.6], $\sigma_{\mathrm{d}}(T)=\sigma_{\mathrm{bb}}(T)$.

Now, we introduce an important property in local spectral theory. The localized version of this property has been introduced by Finch [13], and in the framework of Fredholm theory this property has been characterized in several ways, see Chapter 3 of [1]. $T \in L(X)$ is said to have the single valued extension property at $\lambda_{0} \in \mathbb{C}$ (abbreviated, SVEP at $\lambda_{0}$ ), if for every open disc $\mathbb{D}_{\lambda_{0}} \subseteq \mathbb{C}$ centered at $\lambda_{0}$ the only analytic function $f: \mathbb{D}_{\lambda_{0}} \rightarrow X$ which satisfies the equation

$$
(\lambda I-T) f(\lambda)=0 \quad \text { for all } \lambda \in \mathbb{D}_{\lambda_{0}},
$$

is the function $f \equiv 0$ on $\mathbb{D}_{\lambda_{0}}$. The operator $T$ is said to have SVEP if $T$ has the SVEP at every point $\lambda \in \mathbb{C}$. Evidently, $T \in L(X)$ has SVEP at every point of the resolvent $\rho(T):=\mathbb{C} \backslash \sigma(T)$. Moreover, from the identity theorem for analytic functions it is easily seen that $T$ has SVEP at every point of the boundary $\partial \sigma(T)$ of the spectrum. In particular, $T$ has SVEP at every isolated point of the spectrum. Note that (see [1, Theorem 3.8])

$$
p(\lambda I-T)<\infty \Rightarrow T \text { has SVEP at } \lambda,
$$

and dually

$$
q(\lambda I-T)<\infty \Rightarrow T^{*} \text { has SVEP at } \lambda .
$$

Recall that $T \in L(X)$ is said to be bounded below if $T$ is injective and has closed range. Denote by $\sigma_{\mathrm{ap}}(T)$ the classical approximate point spectrum defined by

$$
\sigma_{\mathrm{ap}}(T):=\{\lambda \in \mathbb{C}: \lambda I-T \text { is not bounded below }\} .
$$

Note that if $\sigma_{\mathrm{s}}(T)$ denotes the surjectivity spectrum

$$
\sigma_{\mathrm{s}}(T):=\{\lambda \in \mathbb{C}: \lambda I-T \text { is not onto }\},
$$

then $\sigma_{\mathrm{ap}}(T)=\sigma_{\mathrm{s}}\left(T^{*}\right), \sigma_{\mathrm{s}}(T)=\sigma_{\mathrm{ap}}\left(T^{*}\right)$ and $\sigma(T)=\sigma_{\mathrm{ap}}(T) \cup \sigma_{\mathrm{s}}(T)$.

It is easily seen from definition of localized SVEP that

$$
\lambda \notin \operatorname{acc} \sigma_{\mathrm{ap}}(T) \Rightarrow T \text { has SVEP at } \lambda,
$$

where acc $\sigma_{\mathrm{ap}}(T)$ means the set of all accumulation points of $\sigma_{\mathrm{ap}}(T)$, and if $T^{*}$ denotes the dual of $T$ then

$$
\lambda \notin \operatorname{acc} \sigma_{\mathrm{s}}(T) \Rightarrow T^{*} \text { has SVEP at } \lambda,
$$


Remark 1.1. The implications (1.1), (1.2), (1.3) and (1.4) are actually equivalences whenever $T \in L(X)$ is semi-Fredholm. Also, $\sigma_{\mathrm{b}}(T)=\sigma_{\mathrm{w}}(T) \cup \operatorname{acc} \sigma(T)$ and $\sigma_{\mathrm{ub}}(T)=\sigma_{\mathrm{uw}}(T) \cup \operatorname{acc} \sigma_{a p}(T)$ (see [1, Chapter 3]).

Denote by iso $K$ the set of all isolated points of $K \subseteq \mathbb{C}$. Let $T \in L(X)$, define

$$
\begin{aligned}
& p_{00}(T)=\sigma(T) \backslash \sigma_{\mathrm{b}}(T), \\
& p_{00}^{a}(T)=\sigma_{\mathrm{ap}}(T) \backslash \sigma_{\mathrm{ub}}(T), \\
& \pi_{00}(T)=\{\lambda \in \operatorname{iso} \sigma(T): 0<\alpha(\lambda I-T)<\infty\}, \\
& \pi_{00}^{a}(T)=\left\{\lambda \in \operatorname{iso} \sigma_{\mathrm{ap}}(T): 0<\alpha(\lambda I-T)<\infty\right\},
\end{aligned}
$$

Observe that, for every $T \in L(X)$, we have $p_{00}(T) \subseteq \pi_{00}(T) \subseteq \pi_{00}^{a}(T)$.

In the sequel we need the following basic results.

Lemma 1.2. For $T \in L(X)$, we have the following statements:

(i) If $p(T)$ and $q(T)$ are both finite, then $p(T)=q(T)$;

(ii) If $p(T)$ and $q(T)$ are both finite, then $\alpha(T)=\beta(T)$;

(iii) If $\alpha(T)=\beta(T)<\infty$, and $p(T)$ or $q(T)$ is finite, then $p(T)=q(T)$.

Proof. A proof of (i) may be found in [15, Prop. 38.3]. For (ii) and (iii), see [15, Prop. 38.6].

Lemma 1.3. If $T \in L(X)$ and $p=p(T)<\infty$, then the following statements are equivalent:

(i) There exists $n \geq p+1$ such that $T^{n}(X)$ is closed;

(ii) $T^{n}(X)$ is closed for all $n \geq p$.

Proof. Define $c_{i}^{\prime}(T)=\operatorname{dim}\left(N\left(T^{i}\right) / N\left(T^{i+1}\right)\right)$. Clearly, $p=p(T)<\infty$ entails that $c_{i}^{\prime}(T)=0$ for all $i \geq p$, so $k_{i}(T)=c_{i}^{\prime}(T)-c_{i+1}^{\prime}(T)=0$ for all $i \geq p$. The equivalence easily follows from [16, Lemma 12].

Lemma 1.4. [11, Lemma 2.1]. Let $T \in L(X)$ and $T_{n}, n \in \mathbb{N}$, be the restriction of the operator $T$ on the subspace $R\left(T^{n}\right)=T^{n}(X)$. Then for all $\lambda \neq 0$, we have:

(i) $N\left(\left(\lambda I-T_{n}\right)^{m}\right)=N\left((\lambda I-T)^{m}\right)$, for any $m$;

(ii) $R\left(\left(\lambda I-T_{n}\right)^{m}\right)=R\left((\lambda I-T)^{m}\right) \cap R\left(T^{n}\right)$, for any $m$;

(iii) $\alpha\left(\lambda I-T_{n}\right)=\alpha(\lambda I-T)$;

(iv) $p\left(\lambda I-T_{n}\right)=p(\lambda I-T)$;

(v) $\beta\left(\lambda I-T_{n}\right)=\beta(\lambda I-T)$.

Lemma 1.5. If $R\left(T^{n}\right)$ is closed in $X$ and $R\left(\left(\lambda I-T_{n}\right)^{m}\right)$ is closed in $R\left(T^{n}\right)$ for $\lambda \neq 0$, then $R\left((\lambda I-T)^{m}\right)$ is closed in $X$.

Proof. If $\lambda \neq 0$ and $R\left(\left(\lambda I-T_{n}\right)^{m}\right)$ is a closed subspace of $R\left(T^{n}\right)$, since $R\left(T^{n}\right)$ is closed in $X$, we have that $R\left(\left(\lambda I-T_{n}\right)^{m}\right)$ is closed in $X$. But, from the incise (ii) in the Lemma 1.4, $R\left(\left(\lambda I-T_{n}\right)^{m}\right)=R\left((\lambda I-T)^{m}\right) \cap R\left(T^{n}\right)$. Thus $R((\lambda I-$ $\left.T)^{m}\right) \cap R\left(T^{n}\right)$ is closed in $X$. Also, if $\lambda \neq 0$, the polynomials $(\lambda-z)^{m}$ and $z^{n}$ have not common divisors, so there exist two polynomials $u$ and $v$ such that $1=(\lambda-z)^{m} u(z)+z^{n} v(z)$, for all $z \in \mathbb{C}$. Hence $I=(\lambda I-T)^{m} u(T)+T^{n} v(T)$ and so $R\left((\lambda I-T)^{m}\right)+R\left(T^{n}\right)=X$. Since both $R\left((\lambda I-T)^{m}\right)$ and $R\left(T^{n}\right)$ are 
paraclosed subspaces, and $R\left((\lambda I-T)^{m}\right) \cap R\left(T^{n}\right)$ and $R\left((\lambda I-T)^{m}\right)+R\left(T^{n}\right)$ are closed, using the Neubauer Lemma [17, Prop. 2.1.2], we have that $R\left((\lambda I-T)^{m}\right)$ is closed.

Recall that for an operator $T \in L(X), 0<p(\lambda I-T)=q(\lambda I-T)<\infty$ precisely when $\lambda$ is a pole of the resolvent of $T$ (see [15, Prop. 50.2]). Also, every pole of the resolvent of $T$ is an isolated point of $\sigma(T)$.

Lemma 1.6. If 0 is not a pole of the resolvent of $T \in L(X)$ and $R\left(T^{n}\right)$ is closed, then

(i) $\pi_{00}(T) \subseteq \pi_{00}\left(T_{n}\right)$;

(ii) $\pi_{00}^{a}(T) \subseteq \pi_{00}^{a}\left(T_{n}\right)$;

Proof. The proof of (i) may be found in [11]. For (ii), see [12].

In the next definition, we describe several spectral properties introduced recently (see [8], [9], [14], [18], [19], [20] and [21]).

Definition 1.7. An operator $T \in L(X)$ is said to satisfy property:

(i) $(w)$, if $\sigma_{a p}(T) \backslash \sigma_{\mathrm{uw}}(T)=\pi_{00}(T)([18])$;

(ii) $(a w)$, if $\sigma(T) \backslash \sigma_{\mathrm{w}}(T)=\pi_{00}^{a}(T)([8])$;

(iii) $(b)$, if $\sigma_{\mathrm{ap}}(T) \backslash \sigma_{\mathrm{uw}}(T)=p_{00}(T)([9])$;

(iv) $(a b)$, if $\sigma(T) \backslash \sigma_{\mathrm{w}}(T)=p_{00}^{a}(T)([8])$;

(v) $(z)$ if $\sigma(T) \backslash \sigma_{\mathrm{uw}}(T)=\pi_{00}^{a}(T)([21])$;

(vi) $(a z)$, if $\sigma(T) \backslash \sigma_{\mathrm{uw}}(T)=p_{00}^{a}(T)([21])$;

(vii) $(v)$, if $\sigma(T) \backslash \sigma_{\mathrm{uw}}(T)=\pi_{00}(T)([20])$;

Also, $T$ is said to satisfy:

(viii) Browder's theorem, if $\sigma_{\mathrm{w}}(T)=\sigma_{\mathrm{b}}(T)([14])$;

(ix) $a$-Browder's theorem, if $\sigma_{\mathrm{uw}}(T)=\sigma_{\mathrm{ub}}(T)([19])$;

(x) generalized Browder's theorem, if $\sigma_{\mathrm{bw}}(T)=\sigma_{\mathrm{bb}}(T)([14])$.

Specific spectral properties have been studied by several authors, through restrictions ([11], [12]) and extensions ([3], [4]). In this paper we show that for a linear operator $T \in L(X)$ acting on a Banach space $X$, all spectral properties in Definition 1.7 are essentially the same for $T$ and some restriction $T_{n}$.

\section{Relations Between the spectra of $T$ And $T_{n}$}

We begin by examining some relations between the spectra of $T$ and $T_{n}$.

Lemma 2.1. Let $T \in L(X)$ and $T_{n}, n \in \mathbb{N}$, be the restriction of the operator $T$ on the subspace $R\left(T^{n}\right)$. If $R\left(T^{n}\right)$ is closed, then:

(i) $\sigma\left(T_{n}\right) \subseteq \sigma(T)$ and $\sigma_{\text {ap }}\left(T_{n}\right) \subseteq \sigma_{\text {ap }}(T)$;

(ii) $\sigma_{\mathrm{w}}\left(T_{n}\right) \subseteq \sigma_{\mathrm{w}}(T)$ and $\sigma_{\mathrm{uw}}\left(T_{n}\right) \subseteq \sigma_{\mathrm{uw}}(T)$;

(iii) $\sigma_{\mathrm{b}}\left(T_{n}\right) \subseteq \sigma_{\mathrm{b}}(T)$ and $\sigma_{\mathrm{ub}}\left(T_{n}\right) \subseteq \sigma_{\mathrm{ub}}(T)$.

Proof. (i) By Lemma 1.4, $\sigma\left(T_{n}\right) \backslash\{0\}=\sigma(T) \backslash\{0\}$. Also, $0 \notin \sigma(T)$ implies $T$ bijective, thus $T=T_{n}$. Then $0 \notin \sigma(T)=\sigma\left(T_{n}\right)$. Consequently $0 \notin \sigma(T)$ implies $0 \notin \sigma\left(T_{n}\right)$, or equivalently, $0 \in \sigma\left(T_{n}\right)$ implies $0 \in \sigma(T)$. Hence $\sigma\left(T_{n}\right) \subseteq \sigma(T)$. For the other inclusion, if $\lambda \notin \sigma_{\text {ap }}(T)$ then $\lambda I-T$ is injective and $R(\lambda I-T)$ is 
closed. Now, here we consider two different cases $\lambda \neq 0$ and $\lambda=0$. If $\lambda \neq 0$, by Lemma 1.4, $N\left(\lambda I-T_{n}\right)=N(\lambda I-T)$ and $R\left(\lambda I-T_{n}\right)=R(\lambda I-T) \cap R\left(T^{n}\right)$ is closed. Hence $\lambda I-T_{n}$ is bounded below, and so $\lambda \notin \sigma_{\text {ap }}\left(T_{n}\right)$. In the other case, $-T$ bounded below implies that $0=p(T)=p\left(T_{n}\right)$ and $R(T)$ is closed. Thus $T_{n}$ is injective and, by Lemma $1.3, R\left(T_{n}\right)=R\left(T^{n+1}\right)$ is closed. From this we obtain that $T_{n}$ is bounded below. Consequently, $\sigma_{\text {ap }}\left(T_{n}\right) \subseteq \sigma_{\text {ap }}(T)$.

(ii) By Lemma 1.4, $\sigma_{\mathrm{w}}\left(T_{n}\right) \backslash\{0\}=\sigma_{\mathrm{w}}(T) \backslash\{0\}$. Also, $0 \notin \sigma_{\mathrm{w}}(T)$ implies $T$ Weyl, thus $T$ is Fredholm and ind $T=0$. Then $T_{0}=T$ is a Fredholm operator, it follows from [6, Proposition 2.1] that also $T_{n}$ Fredholm and ind $T_{n}=\operatorname{ind} T_{0}=0$. But this implies $T_{n}$ is a Weyl operator, thus $0 \notin \sigma_{\mathrm{w}}\left(T_{n}\right)$. This proves that $\sigma_{\mathrm{w}}\left(T_{n}\right) \subseteq \sigma_{\mathrm{w}}(T)$. For the other inclusion, by Lemma 1.4, $\sigma_{\text {uw }}\left(T_{n}\right) \backslash\{0\}=\sigma_{\text {uw }}(T) \backslash\{0\}$. Now, suppose $0 \notin \sigma_{\text {uw }}(T)$. Then $T$ is upper semi-Weyl, thus $T$ is upper semi-Fredholm and ind $T \leq 0$. Again, by [6, Proposition 2.1], $T_{m}$ is upper semi-Fredholm and ind $T_{m}=\operatorname{ind} T_{0}$ for all $m \geq 0$. In particular, $T_{n}$ is upper semi-Weyl. Consequently, $\sigma_{\text {uw }}\left(T_{n}\right) \subseteq \sigma_{\text {uw }}(T)$.

(iii) Follows from (i), (ii) and the spectral equalities of the Remark 1.1.

In general, almost nothing can be said concerning the equality between the spectra of $T$ and $T_{n}$. However, assuming some special conditions, the spectrum, the Browder spectrum and the approximate point spectrum are the same for $T$ and $T_{n}$. Also, $\lambda$ is said to be a left pole of the resolvent of $T \in L(X)$, if $\lambda \in \sigma_{\mathrm{ap}}(T)$ and $\lambda I-T$ left Drazin invertible (see [7]).

Lemma 2.2. Let $T \in L(X)$ and $T_{n}, n \in \mathbb{N}$, be the restriction of the operator $T$ on the subspace $R\left(T^{n}\right)$. If $R\left(T^{n}\right)$ is closed, we have:

(i) If $q(T)=\infty$, then $\sigma\left(T_{n}\right)=\sigma(T)$;

(ii) If 0 is not a pole of the resolvent of $T$, then $\sigma_{\mathrm{b}}\left(T_{n}\right)=\sigma_{\mathrm{b}}(T)$;

(iii) If 0 is not a left pole of the resolvent of $T$, then $\sigma_{\mathrm{ap}}\left(T_{n}\right)=\sigma_{\mathrm{ap}}(T)$.

Proof. (i) By Lemma 1.4, $\sigma\left(T_{n}\right) \backslash\{0\}=\sigma(T) \backslash\{0\}$. Also, $q(T)=\infty$ implies $R(X) \neq X$ and $R\left(T_{n}\right)=R\left(T^{n+1}\right) \neq R\left(T^{n}\right)$, thus $0 \in \sigma(T)$ and $0 \in \sigma\left(T_{n}\right)$. Then $\sigma\left(T_{n}\right)=\sigma(T)$.

(ii) By Lemma 1.4, $\sigma_{\mathrm{b}}\left(T_{n}\right) \backslash\{0\}=\sigma_{\mathrm{b}}(T) \backslash\{0\}$. Now suppose $0 \notin \sigma_{\mathrm{b}}(T)$. Then $T$ is a Browder operator and both $p(T), q(T)$ are finite. By [15, Proposition 38.6], $0<p(T)=q(T)<\infty$, so we have a contradiction. Thus $0 \notin \sigma_{\mathrm{b}}(T)$. On the other hand, $0 \notin \sigma_{\mathrm{b}}\left(T_{n}\right)$ implies $T_{n}$ is a Browder, then $0<p\left(T_{n}\right)=q\left(T_{n}\right)<\infty$. By Lemmas 2 and 3 in [10] and [15, Proposition 38.6], $0<p(T)=q(T)<\infty$, so again we have a contradiction. Therefore $0 \in \sigma_{\mathrm{b}}(T)$ and $0 \in \sigma_{\mathrm{b}}\left(T_{n}\right)$. Consequently, $\sigma_{\mathrm{b}}(T)=\sigma_{\mathrm{b}}\left(T_{n}\right)$.

(iii) By Lemmas 1.4 and 1.5, $\sigma_{\text {ap }}\left(T_{n}\right) \backslash\{0\}=\sigma_{\text {ap }}(T) \backslash\{0\}$. On the other hand, $0 \notin \sigma_{\text {ap }}\left(T_{n}\right)$ implies $p\left(T_{n}\right)=0$, by Lemma $1.3, R\left(T^{n+k}\right)=R\left(\left(T_{n}\right)^{k}\right)$ is closed for all $k \geq 0$. Also, by Lemma 2 in [10], $p(T)<\infty$ because $p\left(T_{n}\right)=0$. Thus, if 
$0 \in \sigma_{\text {ap }}(T)$ then 0 is a left pole of the resolvent of $T$, a contradiction. Hence, $0 \notin \sigma_{\text {ap }}(T)$. Similarly, $0 \notin \sigma_{\text {ap }}(T)$ implies $0 \notin \sigma_{\text {ap }}\left(T_{n}\right)$.

Similarly as in Lemma 1.6, we have the following relations.

Lemma 2.3. Let $T \in L(X)$ and $R\left(T^{n}\right)$ is closed, then

(i) If 0 is not a pole of the resolvent of $T, p_{00}(T) \subseteq p_{00}\left(T_{n}\right)$;

(ii) If 0 is not a left pole of the resolvent of $T, p_{00}^{a}(T) \subseteq p_{00}^{a}\left(T_{n}\right)$.

Proof. (i) Let $\lambda \in p_{00}(T)=\sigma(T) \backslash \sigma_{\mathrm{b}}(T)$, we have that $0<p(\lambda I-T)=q(\lambda I-$ $T)<\infty$. From this equality, and by hypothesis, $\lambda \neq 0$. Hence $\lambda \in \sigma(T) \backslash\{0\}=$ $\sigma\left(T_{n}\right) \backslash\{0\} \subseteq \sigma\left(T_{n}\right)$. On the other hand, by Lemma 2.2, $\lambda \notin \sigma_{\mathrm{b}}(T)=\sigma_{\mathrm{b}}\left(T_{n}\right)$. Consequently, $\lambda \in \sigma\left(T_{n}\right) \backslash \sigma_{\mathrm{b}}\left(T_{n}\right)=p_{00}\left(T_{n}\right)$.

(ii) Let $\lambda \in p_{00}^{a}(T)=\sigma_{\text {ap }}(T) \backslash \sigma_{\mathrm{ub}}(T)$, we have that $\lambda \in \sigma_{\mathrm{ap}}(T), 0<p(\lambda I-T)<$ $\infty$ and $(\lambda I-T)^{k}(X)$ is closed for all $k \in \mathbb{N}$. Then $\lambda$ is a left pole of the resolvent of $T$, and by hypothesis, $\lambda \neq 0$. Thus, $\lambda \in \sigma_{\text {ap }}(T) \backslash\{0\}=\sigma_{\text {ap }}\left(T_{n}\right) \backslash\{0\} \subseteq$ $\sigma_{\mathrm{ap}}\left(T_{n}\right)$. Also, by Lemma 2.2, $\lambda \notin \sigma_{\mathrm{ub}}(T) \supseteq \sigma_{\mathrm{ub}}\left(T_{n}\right)$. Hence $\lambda \notin \sigma_{\mathrm{ub}}\left(T_{n}\right)$ and so $\lambda \in \sigma_{\mathrm{ap}}\left(T_{n}\right) \backslash \sigma_{\mathrm{ub}}\left(T_{n}\right)=p_{00}^{a}\left(T_{n}\right)$. Therefore $p_{00}^{a}(T) \subseteq p_{00}^{a}\left(T_{n}\right)$.

\section{Spectral properties And Restrictions}

In this section we show that the properties studied in section one are essentially the same for $T$ and some restriction $T_{n}$ of $T$ on $R\left(T^{n}\right)$.

Theorem 3.1. If $T \in L(X)$ have ascent infinite, then:

(i) there exists $n \in \mathbb{N}$ such that $R\left(T^{n}\right)$ is closed and $T_{n}$ satisfies property $(w)$ if and only if $T$ satisfies property $(w)$;

(ii) there exists $n \in \mathbb{N}$ such that $R\left(T^{n}\right)$ is closed and $T_{n}$ satisfies property $(b)$ if and only if $T$ satisfies property $(b)$;

(iii) there exists $n \in \mathbb{N}$ such that $R\left(T^{n}\right)$ is closed and $T_{n}$ satisfies Browder's $a$-theorem if and only if $T$ satisfies Browder's a-theorem.

Proof. (i) Assume that $R\left(T^{n}\right)$ is closed and $T_{n}$ satisfies property $(w)$. Let $\lambda \in$ $\pi_{00}(T)$, by Lemma 1.6, $\lambda \in \pi_{00}(T) \subseteq \pi_{00}\left(T_{n}\right)=\sigma_{\text {ap }}\left(T_{n}\right) \backslash \sigma_{\text {uw }}\left(T_{n}\right)$. Since $\lambda I-T_{n}$ is a semi-Fredholm operator and $\lambda \in$ iso $\sigma\left(T_{n}\right)$, then $\lambda I-T_{n}$ has both ascent and descent finite. Thus $0<p\left(\lambda I-T_{n}\right)=q\left(\lambda I-T_{n}\right)<\infty$. From this equality, and by hypothesis, if $\lambda=0$ we have that $0<p\left(T_{n}\right)=q\left(T_{n}\right)<\infty$. By Lemmas 2 and 3 in [10] and [15, Proposition 38.6], $0<p(T)=q(T)<\infty$, a contradiction. But $0<\alpha\left(\lambda I-T_{n}\right)=\beta\left(\lambda I-T_{n}\right)<\infty$, because $0<p\left(\lambda I-T_{n}\right)=q\left(\lambda I-T_{n}\right)<\infty$. Now, being $\lambda \neq 0$, by Lemma 1.4

$$
0<\beta(\lambda I-T)=\beta\left(\lambda I-T_{n}\right)=\alpha\left(\lambda I-T_{n}\right)=\alpha(\lambda I-T)<\infty,
$$

also $p(\lambda I-T)=p\left(\lambda I-T_{n}\right)<\infty$, then $0<p(\lambda I-T)=q(\lambda I-T)<\infty$. Consequently $\lambda \in \sigma_{\text {ap }}(T) \backslash \sigma_{\text {uw }}(T)$. Hence, $\pi_{00}(T) \subseteq \sigma_{\text {ap }}(T) \backslash \sigma_{\text {uw }}(T)$. For the reverse inclusion, observe that if $\lambda \in \sigma_{\mathrm{ap}}(T) \backslash \sigma_{\mathrm{uw}}(T)$, by hypothesis and the Lemmas 2.1 and 2.2, we have that $\lambda \in \sigma_{\text {ap }}\left(T_{n}\right) \backslash \sigma_{\text {uw }}\left(T_{n}\right)=\pi_{00}\left(T_{n}\right)$. From this equality and proceding as in the first part, we easily obtain the equality $0<p(\lambda I-T)=q(\lambda I-T)<\infty$, which implies that $\lambda \in \pi_{00}(T)$. Thus, 
$\sigma_{\text {ap }}(T) \backslash \sigma_{\text {uw }}(T) \subseteq \pi_{00}(T)$. Consequently, $\sigma_{\text {ap }}(T) \backslash \sigma_{\text {uw }}(T)=\pi_{00}(T)$ and $T$ satisfies property $(w)$.

(ii) Suppose that property (b) holds for $T_{n}$. Let $\lambda \in p_{00}(T)$, by Lemma 1.6, $\lambda \in p_{00}(T) \subseteq p_{00}\left(T_{n}\right)=\sigma_{\mathrm{ap}}\left(T_{n}\right) \backslash \sigma_{\mathrm{uw}}\left(T_{n}\right)$. Then $\lambda I-T_{n}$ is a semi-Fredholm operator and both $p\left(\lambda I-T_{n}\right)$ and $q\left(\lambda I-T_{n}\right)$ are finite. Similarly, as in the proof of part (i), we obtain the equality $0<p(\lambda I-T)=q(\lambda I-T)<\infty$ and hence $\lambda \in \sigma_{\text {ap }}(T) \backslash \sigma_{\text {uw }}(T)$. Thus $p_{00}(T) \subseteq \sigma_{\text {ap }}(T) \backslash \sigma_{\text {uw }}(T)$. For the other inclusion, suppose that $\lambda \in \sigma_{\text {ap }}(T) \backslash \sigma_{\mathrm{uw}}(T)$. By the hypothesis and the Lemmas 2.1 and 2.2, $\lambda \in \sigma_{\text {ap }}\left(T_{n}\right) \backslash \sigma_{\text {uw }}\left(T_{n}\right)=p_{00}\left(T_{n}\right)$. From this equality and proceding as above, we obtain that $0<p(\lambda I-T)=q(\lambda I-T)<\infty$. Thus $\lambda \in p_{00}(T)$. Therefore, $\sigma_{\text {ap }}(T) \backslash \sigma_{\text {uw }}(T) \subseteq p_{00}(T)$. Consequently, $\sigma_{\text {ap }}(T) \backslash \sigma_{\text {uw }}(T)=p_{00}(T)$, and $T$ satisfies property $(b)$.

(iii) If $T_{n}$ satisfies $a$-Browder's theorem, then iso $\sigma_{\text {ap }}\left(T_{n}\right) \subseteq \sigma_{\text {uw }}\left(T_{n}\right)$. By Lemmas 2.1 and 2.2, we have iso $\sigma_{\text {ap }}(T)=$ iso $\sigma_{\text {ap }}\left(T_{n}\right) \subseteq \sigma_{\text {uw }}\left(T_{n}\right) \subseteq \sigma_{\text {uw }}(T)$. Thus, $\sigma_{\mathrm{ub}}(T)=\sigma_{\mathrm{uw}}(T) \cup$ iso $\sigma_{\mathrm{ap}}(T)=\sigma_{\mathrm{uw}}(T)$ and hence $T$ satisfies $a$-Browder's theorem.

For the converse of all these implications. Observe that for $n=0$, trivially $R\left(T^{0}\right)=X$ is closed and $T_{0}=T$.

Theorem 3.2. If $T \in L(X)$ have descent infinite, then:

(i) there exists $n \in \mathbb{N}$ such that $R\left(T^{n}\right)$ is closed and $T_{n}$ satisfies property (aw) if and only if $T$ satisfies property (aw);

(ii) there exists $n \in \mathbb{N}$ such that $R\left(T^{n}\right)$ is closed and $T_{n}$ satisfies property $(a b)$ if and only if $T$ satisfies property $(a b)$;

(iii) there exists $n \in \mathbb{N}$ such that $R\left(T^{n}\right)$ is closed and $T_{n}$ satisfies property $(v)$ if and only if $T$ satisfies property $(v)$;

(iv) there exists $n \in \mathbb{N}$ such that $R\left(T^{n}\right)$ is closed and $T_{n}$ satisfies Browder's theorem if and only if $T$ satisfies Browder's theorem.

(v) there exists $n \in \mathbb{N}$ such that $R\left(T^{n}\right)$ is closed and $T_{n}$ satisfies generalized Browder's theorem if and only if T satisfies generalized Browder's theorem.

Proof. (i) Suppose that $R\left(T^{n}\right)$ is closed and $T_{n}$ satisfies property (aw). Let $\lambda \in$ $\pi_{00}^{a}(T)$, by Lemma 1.6, $\lambda \in \pi_{00}^{a}(T) \subseteq \pi_{00}^{a}\left(T_{n}\right)=\sigma\left(T_{n}\right) \backslash \sigma_{\mathrm{w}}\left(T_{n}\right)$. Since $\lambda I-T_{n}$ is a Fredholm operator, ind $\left(\lambda I-T_{n}\right)=0$ and $\lambda \in$ iso $\sigma_{\text {ap }}\left(T_{n}\right)$. Then $p\left(\lambda I-T_{n}\right)<\infty$ and $0<\alpha\left(\lambda I-T_{n}\right)=\beta\left(\lambda I-T_{n}\right)<\infty$, thus $0<p\left(\lambda I-T_{n}\right)=q\left(\lambda I-T_{n}\right)<\infty$. From this equality, and by hypothesis, $\lambda=0$ implies that $0<p\left(T_{n}\right)=q\left(T_{n}\right)<\infty$. By Lemmas 2 and 3 in [10] and [15, Proposition 38.6], $0<p(T)=q(T)<\infty$, a contradiction. Now, being $\lambda \neq 0$, by Lemma 1.4

$$
0<\beta(\lambda I-T)=\beta\left(\lambda I-T_{n}\right)=\alpha\left(\lambda I-T_{n}\right)=\alpha(\lambda I-T)<\infty
$$

But $p(\lambda I-T)=p\left(\lambda I-T_{n}\right)<\infty$, then $0<p(\lambda I-T)=q(\lambda I-T)<\infty$. Consequently $\lambda \in \sigma(T) \backslash \sigma_{\mathrm{w}}(T)$. Hence, $\pi_{00}^{a}(T) \subseteq \sigma(T) \backslash \sigma_{\mathrm{w}}(T)$. For the reverse inclusion, observe that if $\lambda \in \sigma(T) \backslash \sigma_{\mathrm{w}}(T)$, by hypothesis and Lemmas 2.1 and 2.2, we have that $\lambda \in \sigma\left(T_{n}\right) \backslash \sigma_{\mathrm{w}}\left(T_{n}\right)=\pi_{00}^{a}\left(T_{n}\right)$. From this equality and preceding 
as in the first part, we easily obtain the equality $0<p(\lambda I-T)=q(\lambda I-T)<\infty$, which implies that $\lambda \in \pi_{00}^{a}(T)$. Thus, $\sigma(T) \backslash \sigma_{\mathrm{w}}(T) \subseteq \pi_{00}^{a}(T)$. Consequently, $\sigma(T) \backslash \sigma_{\mathrm{w}}(T)=\pi_{00}^{a}(T)$ and $T$ satisfies property $(a w)$.

(ii) Suppose that $R\left(T^{n}\right)$ is closed and $T_{n}$ satisfies property $(a b)$. Let $\lambda \in p_{00}^{a}(T)$, by Lemma 1.6, $\lambda \in p_{00}^{a}(T) \subseteq p_{00}^{a}\left(T_{n}\right)=\sigma\left(T_{n}\right) \backslash \sigma_{\mathrm{w}}\left(T_{n}\right)$. Since $\lambda I-T_{n}$ is a Fredholm operator, ind $\left(\lambda I-T_{n}\right)=0$ and $p\left(\lambda I-T_{n}\right)<\infty$. Then $p\left(\lambda I-T_{n}\right)<\infty$ and $0<\alpha\left(\lambda I-T_{n}\right)=\beta\left(\lambda I-T_{n}\right)<\infty$, thus $0<p\left(\lambda I-T_{n}\right)=q\left(\lambda I-T_{n}\right)<\infty$. By using the same argument of part (i), we obtain the equality $0<p(\lambda I-T)=$ $q(\lambda I-T)<\infty$, which implies that $\lambda \in \sigma(T) \backslash \sigma_{\mathrm{w}}(T)$. Thus $p_{00}^{a}(T) \subseteq \sigma(T) \backslash \sigma_{\mathrm{w}}(T)$. For the reverse inclusion observe that if $\lambda \in \sigma(T) \backslash \sigma_{\mathrm{w}}(T)$, by hypothesis and Lemmas 2.1 and 2.2, we have that $\lambda \in \sigma\left(T_{n}\right) \backslash \sigma_{\mathrm{w}}\left(T_{n}\right)=p_{00}^{a}\left(T_{n}\right)$. As above, it then follows that $0<p(\lambda I-T)=q(\lambda I-T)<\infty$ and hence $\lambda \in p_{00}^{a}(T)$. Thus, $\sigma(T) \backslash \sigma_{\mathrm{w}}(T) \subseteq p_{00}^{a}(T)$. Consequently, $\sigma(T) \backslash \sigma_{\mathrm{w}}(T)=p_{00}^{a}(T)$ and $T$ satisfies property $(a b)$.

(iii) Suppose that $T_{n}$ satisfies property $(v)$. Let $\lambda \in \pi_{00}(T)$, by Lemma 1.6, $\lambda \in \pi_{00}(T) \subseteq \pi_{00}\left(T_{n}\right)=\sigma\left(T_{n}\right) \backslash \sigma_{\mathrm{uw}}\left(T_{n}\right)$. Then $\lambda I-T_{n}$ is a semi-Fredholm operator and $\lambda I-T_{n}$ has both ascent and descent finite. By using the same argument of Theorem 3.1, we deduce that $0<p(\lambda I-T)=q(\lambda I-T)<\infty$. Then $\lambda \in \sigma(T) \backslash \sigma_{\mathrm{uw}}(T)$, thus $\pi_{00}(T) \subseteq \sigma(T) \backslash \sigma_{\mathrm{uw}}(T)$. For the reverse inclusion observe that if $\lambda \in \sigma(T) \backslash \sigma_{\text {uw }}(T)$, by hypothesis and Lemmas 2.1 and 2.2, we have that $\lambda \in \sigma\left(T_{n}\right) \backslash \sigma_{\text {uw }}\left(T_{n}\right)=\pi_{00}\left(T_{n}\right)$. As above, it then follows that $0<p(\lambda I-T)=q(\lambda I-T)<\infty$ and hence $\lambda \in \pi_{00}(T)$. Thus, $\sigma(T) \backslash \sigma_{\text {uw }}(T) \subseteq \pi_{00}(T)$. Consequently, $\sigma(T) \backslash \sigma_{\text {uw }}(T)=\pi_{00}(T)$ and $T$ satisfies property $(v)$.

(iv) If $T_{n}$ satisfies Browder's theorem, then iso $\sigma\left(T_{n}\right) \subseteq \sigma_{\mathrm{w}}\left(T_{n}\right)$. By Lemmas 2.1 and 2.2, we have iso $\sigma(T)=$ iso $\sigma\left(T_{n}\right) \subseteq \sigma_{\mathrm{w}}\left(T_{n}\right) \subseteq \sigma_{\mathrm{w}}(T)$. Thus, $\sigma_{\mathrm{b}}(T)=\sigma_{\mathrm{w}}(T) \cup$ iso $\sigma(T)=\sigma_{\mathrm{w}}(T)$ and hence $T$ satisfies Browder's theorem.

(v) It follows from (iv) and the equivalence between Browder's theorem and generalized Browder's theorem proved in [2].

For the converse of all these implications. Observe that for $n=0$, trivially $R\left(T^{0}\right)=X$ is closed and $T_{0}=T$.

Theorem 3.3. If $T \in L(X)$ has both ascent and descent infinite, then:

(i) there exists $n \in \mathbb{N}$ such that $R\left(T^{n}\right)$ is closed and $T_{n}$ satisfies property $(z)$ if and only if $T$ satisfies property $(z)$;

(ii) there exists $n \in \mathbb{N}$ such that $R\left(T^{n}\right)$ is closed and $T_{n}$ satisfies property (az) if and only if $T$ satisfies property $(a z)$;

(iii) there exists $n \in \mathbb{N}$ such that $R\left(T^{n}\right)$ is closed and $T_{n}$ satisfies property $(a b)$ if and only if $T$ satisfies property $(a b)$.

Proof. The proof is analogous to the Theorems 3.1 and 3.2. 
We give one illustrative example for the behavior of an operator $T$ and its restrictions $T_{n}$, when both $p(T)$ and $q(T)$ are finite.

Example 3.4. Let $X$ be a Banach space, and assume that $Y$ and $Z$ are proper closed subspaces of $X$ with $X=Y \oplus Z$. Let $T$ be the projection of $X$ on $Y$ which is zero on $Z$. Since $T$ is a projection operator, i.e $T^{2}=T$, then $p(T)<\infty, q(T)<\infty$ and $\sigma(T)=\{0,1\}$. Also, the operator $T_{n}=\left.T\right|_{R\left(T^{n}\right)}$ is the identity operator on $Y$ for all $n \geq 1$. Thus $\sigma\left(T_{n}\right)=\{1\}$, for all $n \geq 1$. Assuming that neither $Y$ nor $Z$ is finite dimensional, then both $T$ and $T_{n}$ satisfy the properties given in Definition 1.7. Now, if $Y$ is infinite dimensional and $Z$ is finite dimensional, then $T_{n}$ satisfy the properties given in Definition 1.7, for all $n \geq 1$. But, $T$ does not satisfy the properties (i), (ii), (v) and (vii) in Definition 1.7.

Remark 3.5. There are more alternative ways to express Theorem 3.1 (resp. 3.2). We may replace the assumption $T$ have ascent infinite (resp. have descent infinite) by $T$ does not have SVEP at 0 (resp. $T^{*}$ does not have SVEP at 0 ).

Acknowledgement. We thank very much the anonymous referee for his/her helpful comments.

\section{REFERENCES}

1. P. Aiena, Fredholm and Local Spectral Theory, with Application to Multipliers, Kluwer Acad. Publishers, 2004.

2. P. Aiena, M.T. Biondi and C. Carpintero, On Drazin invertibility, Proc. Amer. Math. Soc. 136 (2008), 2839-2848.

3. P. Aiena, C. Muneo and Z. Lingling, Weyl's theorems and extensions of bounded linear operators, Tokyo J. Math. (2) 35(2012), 279-289.

4. B. Barnes, The spectral and Fredholm theory of extensions of bounded linear operators, Proc. Amer. Math. Soc. (4) 105(1989), 941-949.

5. M. Berkani, Restriction of an operator to the range of its powers, Studia Math. (2) 140 (2000), 163-175.

6. M. Berkani and M. Sarih, On semi B-Fredholm operators, Glasgow Math. J. 43(2001), $457-465$.

7. M. Berkani and J. Koliha, Weyl type theorems for bounded linear operators, Acta Sci. Math. (Szeged). 69 (2003), 359-376.

8. M. Berkani and H. Zariouh, New extended Weyl type theorems, Mat. Vesnik. 62 (2010), $145-154$.

9. M. Berkani, M. Sarih and H. Zariouh, Browder-type theorems and SVEP, Mediterr. J. Math. 8 (2011), 399-409.

10. C. Carpintero, O. García, E. Rosas and J. Sanabria, B-Browder Spectra an Localized SVEP, Rend. Circ. Mat. Palermo. 57 (2008), 241-255.

11. C. Carpintero, D. Muñoz, E. Rosas, O. García and J. Sanabria, Weyl type theorems and restrictions for bounded linear operators, Extractha Mathematicae. 28 (2013), no. 1, 127139.

12. C. Carpintero, D. Muñoz, E. Rosas, O. García and J. Sanabria, Weyl type theorems and restrictions, Mediterr. J. Math (to appear), doi 10.1007/s00009-013-0369-7.

13. J.K. Finch, The single valued extension property on a Banach space, Pacific J. Math. 58 (1975), 61-69.

14. R.E. Harte and W.Y. Lee, Another note on Weyl's theorem, Trans. Amer. Math. Soc. 349 (1997), 2115-2124.

15. H. Heuser, Functional Analysis, Marcel Dekker, New York 1982. 
16. M. Mbekhta and V. Müller, On the axiomatic theory of the spectrum II, Studia Math. 119 (1996), 129-147.

17. J.P. Labrousse, Les opérateurs quasi Fredholm: une généralization des opérateurs semi Fredholm, Rend. Circ. Mat. Palermo. (2) 29 (1980), no. 2, 161-258.

18. V. Rakočević, Operators obeying a-Weyl's theorem, Rev. Roumaine Math. Pures Appl. 34 (1989), no. 10, 915-919.

19. V. Rakočević, On the essential approximate point spectrum II, Math. Vesnick. 36 (1984), 89-97.

20. J. Sanabria, C. Carpintero, E. Rosas and O. García, On generalized property $(v)$ for bounded linear operators, Studia Math. 212 (2012), 141-154.

21. H. Zariouh, Property (gz) for bounded linear operators, Mat. Vesnik. 65 (2013), no. 1, 94-103

1 Departamento de Matemáticas, Universidad de Oriente, Cumaná, Venezuela and Facultad de Ciencias Básicas, Universidad del Atlántico, Barranquilla, Colombia.

E-mail address: carpintero.carlos@gmail.com

E-mail address: ennisrafael@gmail.com

2 Facultad de Ciencias Básicas, Universidad del Atlántico, Barranquilla, Colombia.

E-mail address: jorge.jrodri@gmail.com

${ }^{3}$ Departamento de Matemáticas, Universidad de Oriente, Cumaná, Venezuela. E-mail address: damupi2001@yahoo.com

E-mail address: alcalakarlapino0612@hotmail.com 